\title{
THE SIGNATURE OF THE FIXED SET OF A MAP OF ODD PERIOD
}

\author{
J. P. ALEXANDER, G. C. HAMRICK AND J. W. VICK ${ }^{1}$
}

\begin{abstract}
Let $T$ be a diffeomorphism of odd period $n$ on a closed smooth manifold $M^{2 k}$. The Conner-Floyd analysis of fixed point data and the Atiyah-Singer Index Theorem are applied to prove there exist methods of orienting the components $F$ of the fixed set of $T$, depending only on $n$, so that $\Sigma_{F} \operatorname{sgn} F \equiv \operatorname{sgn} M \bmod 4$ whenever $T^{*}$ is the identity on $H^{k}(M ; Q)$. Other special results of this type are obtained when assumptions are made restricting the possible eigenvalues in the normal bundle to the fixed set.
\end{abstract}

Let $M^{2 n}$ be a closed oriented differentiable manifold and $T$ an orientation preserving diffeomorphism of $M$ of period $k, k$ an odd integer. Denote by $F$ the fixed point set of $T$. Our principal result is then

THEOREM 1. If $T^{*}$ is the identity on $H^{n}(M ; Q)$, then there is a systematic orientation for $F$ so that

$$
\operatorname{sgn}(M) \equiv \operatorname{sgn}(F) \quad \bmod 4 .
$$

The technique for orienting $F$ depends only on $k$ and not on $[T, M]$. This generalizes considerably Corollary 2.11 of [1], and is the best possible result for manifolds of (positive) dimension $\equiv 0 \bmod 4$. The proof involves the connection between the Atiyah-Singer-Segal $G$-Signature Theorem [2], [3] and the Conner-Floyd computation of $\Re_{*}\left(\mathbf{Z}_{k}\right)$, the bordism algebra of local information for $\mathbf{Z}_{k}$ actions [4], [5].

Denote by $\theta_{*}\left(\mathbf{Z}_{k}\right)$ the bordism ring of orientation preserving actions of $\mathbf{Z}_{k}$ on closed smooth manifolds and by $\mathfrak{\pi}_{*}\left(\mathbf{Z}_{k}\right)$ the bordism ring of actions of $\mathbf{Z}_{k}$ on compact oriented smooth manifolds with boundary, having no fixed points on the boundary. $\Re_{*}\left(\mathbf{Z}_{k}\right)$ may be given a bundle theoretic interpretation [4] as follows: We consider bordism classes $\left[\left(\xi_{1}, \ldots, \xi_{(k-1) / 2}\right) \rightarrow V\right]$ of ordered $(k-1) / 2$-tuples of complex vector bundles over closed oriented manifolds $V$. (We allow 0 as a place holder in case $\xi_{r}$ is the 0 -bundle.) If $\varepsilon:\{1,2, \ldots$, $(k-1) / 2\} \rightarrow\{ \pm 1\}$ is a function and $\mathbf{Z}_{k}$ acts in $\xi_{r}$ by multiplication by $\lambda^{\varepsilon(r) \cdot r}, \lambda=\exp (2 \pi i / k)$, the orientations on the $\left\{\xi_{r}\right\}$ and on $V$ induce an orientation on the disk bundle. The action of $\mathbf{Z}_{k}$ is fixed point free on the sphere bundle so that the disk bundle gives rise to an element of $\mathfrak{N}_{*}\left(\mathbf{Z}_{k}\right)$. In fact this correspondence is an isomorphism of bordism theories.

The homomorphism fix: $\Theta_{*}\left(\mathbf{Z}_{k}\right) \rightarrow \mathfrak{N}_{*}\left(\mathbf{Z}_{k}\right)$ is given by fix $([T, M])$

Received by the editors January 20, 1975 and, in revised form, April 25, 1975.

AMS (MOS) subject classifications (1970). Primary 55C35, 57D99.

1 This research was supported by NSF Grant GP-43775. 
$=\sum_{F}\left[T_{F},\left(N_{F}, \partial N_{F}\right)\right]$ where $N_{F}$ is an invariant normal tube around a component $F$ of the fixed set of $T$ in $M$. There is a splitting of the normal bundle of $F$ into eigenbundles $\left(\xi_{1}, \ldots, \xi_{(k-1) / 2}\right) \rightarrow F$ where the eigenvalue for $\xi_{r}$ is $\lambda^{\varepsilon(r) \cdot r}$ and the orientation on $F$ is induced by the orientations on the $\left\{\xi_{r}\right\}$ and the orientation on $N_{F}$ given by $M$. (Clearly different choices of the function $\varepsilon$ may produce different orientations of $F$.) Thus

$$
\left[T_{F},\left(N_{F}, \partial N_{F}\right)\right]=\left[\left(\xi_{1}, \ldots, \xi_{(k-1) / 2}\right) \rightarrow F\right] .
$$

The $G$-signature homomorphism $\sigma: \dot{\vartheta}_{*}\left(\mathbf{Z}_{k}\right) \rightarrow \mathbf{Z}(\lambda)$ defined by Atiyah and Singer [3] assigns to the bordism class of an action an algebraic integer that depends only on the fixed point data. This leads to a commutative diagram

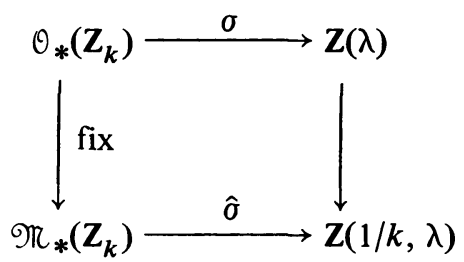

In part, the Atiyah-Singer-Segal $G$-Signature Theorem [2], [3] computes the formula expressing the global invariant $\sigma[T, M]$ in terms of the fixed point information $\hat{\boldsymbol{\sigma}}(\mathrm{fix}[T, M])$. The image of $\hat{\sigma}$ is actually contained in the subring of $\mathbf{Z}(\lambda / k)$ given by

$$
\begin{aligned}
& S=\left\{\sum_{i=1}^{(k-1) / 2} m_{i}\left(\lambda^{i}+\lambda^{-i}\right)+\sum_{i=1}^{(k-1) / 2} n_{i}\left(\lambda^{i}-\lambda^{-i}\right) \mid m_{i} \equiv m_{j} \bmod 2,\right. \\
& \left.n_{i} \equiv n_{j} \bmod 2 \text { and } m_{i}, n_{i} \in \mathbf{Z}(1 / k)\right\}
\end{aligned}
$$

and is generated [4] by 1 and

$$
\left\{c_{r}=\frac{\lambda^{r}+1}{\lambda^{r}-1} \mid 1 \leqslant r \leqslant \frac{k-1}{2}\right\} .
$$

Now suppose $\theta: S \rightarrow \mathbf{Z}_{4}$ is a ring homomorphism such that $\theta(1)$ $=1$ and $\theta\left(c_{r}\right)= \pm 1$ for each $r$. There is a corresponding function $\varepsilon$ : $\{1,2, \ldots,(k-1) / 2\} \rightarrow\{ \pm 1\}$ given by $\varepsilon(r)=\theta\left(c_{r}\right)$. As noted previously such a function $\varepsilon$ gives rise to a systematic choice of eigenvalues in the normal bundle to a component $F$ of the fixed set and hence a well-defined orientation of $F$.

To analyze the composition $\Re_{*}\left(\mathbf{Z}_{k}\right) \stackrel{\hat{\sigma}}{\rightarrow} S \stackrel{\theta}{\rightarrow} \mathbf{Z}_{4}$ we use the fact that $\Re_{*}\left(\mathbf{Z}_{k}\right)$ is a polynomial algebra over $\Omega_{*}^{S O}$ on generators

$$
y_{r, j}=\left[\left(0, \ldots, \xi_{r}, \ldots, 0\right) \rightarrow C P(j)\right]
$$

where $\xi_{r}$ is the conjugate Hopf bundle and $\mathbf{Z}_{k}$ acts in $\xi_{r}$ by $\lambda^{\varepsilon(r) \cdot r}$ [4]. Applying the commutativity of the diagram (2) to the action on $C P(j+1)$ given by $T_{j}\left[z_{0}, \ldots, z_{j+1}\right]=\left[\lambda^{\varepsilon(r) \cdot r} z_{0}, z_{1}, \ldots, z_{j+1}\right]$ we get 


$$
\hat{\boldsymbol{\sigma}}\left(y_{r, j}\right)= \begin{cases}{\left[\hat{\boldsymbol{\sigma}}\left(y_{r, 0}\right)\right]^{j+1},} & j \text { even, } \\ 1-\left[\hat{\boldsymbol{\sigma}}\left(y_{r, 0}\right)\right]^{j+1}, & j \text { odd } .\end{cases}
$$

Now $\hat{\sigma}\left[y_{r, 0}\right]=\varepsilon(r) \cdot c_{r}$ so

$$
\theta\left(\hat{\sigma}\left(y_{r, j}\right)\right)= \begin{cases}1, & j \text { even, } \\ 0, & j \text { odd },\end{cases}
$$

which agrees with sgn $C P(j)$. Therefore the composite $\mathfrak{\Re}_{*}\left(\mathbf{Z}_{k}\right) \stackrel{\hat{\boldsymbol{\theta}}}{\rightarrow} S \stackrel{\theta}{\rightarrow} \mathbf{Z}_{4}$ is given by

$$
\theta \hat{\sigma}\left[\left(\xi_{1}, \ldots, \xi_{(k-1) / 2}\right) \rightarrow V\right] \equiv \operatorname{sgn} V \bmod 4 .
$$

Hence the commutative diagram

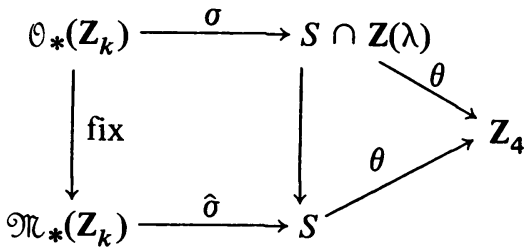

implies that $\theta(\sigma[T, M])=\theta(\hat{\sigma}(\operatorname{fix}[T, M]))=\sum_{F} \operatorname{sgn} F \bmod 4$. When $T^{*}$ is the identity on $H^{n}(M ; Q), \sigma[T, M]=\operatorname{sgn} M$. Thus Theorem 1 follows from

Proposition 3. There are $2^{\varphi(k) / 2}$ distinct ring homomorphisms $\theta: S \rightarrow \mathbf{Z}_{4}$ such that $\theta(1)=1$ and $\theta\left(c_{r}\right)= \pm 1$ for each $r$. (These may not all yield distinct orientations.)

To prove this let $\beta_{i}=2\left(\lambda^{i}+\lambda^{-i}\right), \eta_{j}=2\left(\lambda^{j}-\lambda^{-j}\right)$ and

$$
Y=\sum_{j=1}^{(k-1) / 2} \lambda^{j}-\lambda^{-j}
$$

Then $S$ is generated as a $\mathbf{Z}(1 / k)$ module by

$$
\left\{\beta_{i}\right\}_{i=1}^{(k-1) / 2}, \quad\left\{\eta_{j}\right\}_{j=1}^{(k-1) / 2}, \quad Y \text { and }-1=\sum_{i=1}^{(k-1) / 2} \lambda^{i}+\lambda^{-i} .
$$

The $\mathbf{Z}(1 / k)$-submodule $U$ of $S$ generated by $\left\{\beta_{i}, \eta_{j} \mid 1 \leqslant i, j \leqslant(k-1) / 2\right\}$ is an ideal. If $x \in S$, denote by $\bar{x}$ the image of $x$ in the quotient $S / 2 U$. Let $S_{\mathbf{R}}=S \cap \mathbf{R}$ and $A=S_{\mathbf{R}} / 2 U \cap \mathbf{R}$. As an abelian group $A \simeq \mathbf{Z}_{\mathbf{4}}$ $\oplus\left(\mathbf{Z}_{2}\right)^{(k) / 2-1}$.

Now $A$ is generated by $\overline{1}$ and $\left\{\bar{\beta}_{i}\right\}_{i=1}^{(k-1) / 2}$. Since each $\bar{\beta}_{i}$ has order two and $\bar{\beta}_{i} \bar{\beta}_{j}=0$, it can be shown that any linear map $\hat{\theta}: A \rightarrow \mathbf{Z}_{4}$ such that $\hat{\theta}(\overline{1})=1$ is a ring homomorphism. Hence there are $2^{\varphi(k) / 2-1}$ ring homomorphisms $\hat{\theta}: A \rightarrow \mathbf{Z}_{4}$ with $\hat{\theta}(\overline{1})=1$. Noting that $\bar{Y}^{2}=1$, one easily verifies that each such homomorphism admits two extensions $\hat{\theta}: S / 2 U \rightarrow \mathbf{Z}_{4}$ which send $\bar{Y}$ to +1 and -1 . Thus there are $2^{\varphi(k) / 2}$ ring homomorphisms from $S / 2 U$ to $\mathbf{Z}_{4}$ sending $\overline{1}$ to 1 and $\bar{Y}$ to \pm 1 .

It can be checked that for each $j, c_{j}= \pm Y \bmod U$, so all of the above homomorphisms have $\hat{\theta}\left(\bar{c}_{j}\right)= \pm 1$. For each $\hat{\theta}$ define $\theta$ to be the composition 


$$
S \rightarrow S / 2 U \stackrel{\hat{\theta}}{\rightarrow} \mathbf{Z}_{4} .
$$

This completes the proof of Proposition 3.

For certain special classes of actions there is a stronger relation than that given by Theorem 1. Specifically we have the following theorem.

THEOREM 4. Suppose $T$ is a smooth effective map of odd period $k$ on $M^{2 n}$ such that there is only one type of irreducible representation about the fixed set. Then if $T^{*}$ is the identity on $H^{n}(M ; Q), \operatorname{sgn} M$ is congruent to $\operatorname{sgn} F \bmod 2^{\varphi(k)}$.

Proof. Suppose that $\lambda$ is the eigenvalue corresponding to the one type of irreducible representation. We may as well assume that $\lambda=\exp (2 \pi i / k)$. Let $\psi_{k}(t)=t^{m}+a_{m-1} t^{m-1}+\cdots+a_{0}$ be the cyclotomic polynomial for $\lambda$, where $m=\varphi(k)$. Define

$$
\begin{aligned}
f_{k}(t) & =(t-1)^{m} \psi_{k}((t+1) /(t-1)) \\
& =(t+1)^{m}+a_{m-1}(t+1)^{m-1}(t-1)+\cdots .
\end{aligned}
$$

Note that $f_{k}(1)=2^{\varphi(k)}$ and $f_{k}((\lambda+1) /(\lambda-1))=0$. Also $f_{k}(0)=\psi_{k}(-1)$ $= \pm 1$ so that $f_{k}$ is primitive. Together with the Gauss lemma this implies that the natural homomorphism

$$
\mathbf{Z}[t] /\left\langle f_{k}(t)\right\rangle \rightarrow Q[t] /\left\langle f_{k}(t)\right\rangle
$$

is injective, so we can identify $\mathbf{Z}[t] /\left\langle f_{k}(t)\right\rangle$ with $\mathbf{Z}(x) \subseteq Q(\lambda)$ where $x$ $=(\lambda+1) /(\lambda-1)$. There is also a natural map $\mathbf{Z}[t] /\left\langle f_{k}(t)\right\rangle \rightarrow \mathbf{Z} / 2^{\varphi(k)} \mathbf{Z}$ given by sending $g(t)$ to $g(1)$.

Let $\widetilde{\pi}_{*}\left(\mathbf{Z}_{k}\right)$ denote the subgroup of $\mathfrak{N}_{*}\left(\mathbf{Z}_{k}\right)$ consisting of those actions with the prescribed representation type about the fixed set. Similarly let $\overline{\mathcal{O}}_{*}\left(\mathbf{Z}_{k}\right)$ be the subgroup of $\Theta_{*}\left(\mathbf{Z}_{k}\right)$ consisting of those actions having this representation about the fixed set and further having $T^{*}=$ identity on the middle dimensional rational cohomology.

On $\overline{\mathcal{O}}_{*}\left(\mathbf{Z}_{k}\right)$ we have that $\sigma(T, M)=\operatorname{sgn}(M)$, and $\hat{\sigma}$ restricted to $\bar{\Re}_{*}\left(\mathbf{Z}_{k}\right)$ takes values in $\mathbf{Z}(x)$ where $x=(\lambda+1) /(\lambda-1)$. Hence we have a commutative diagram

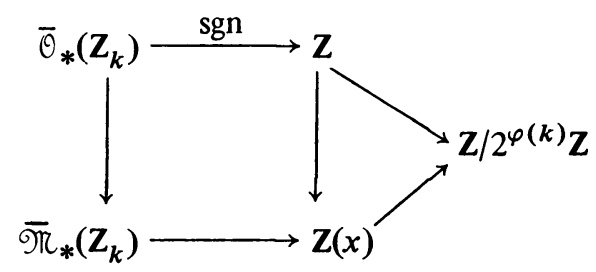

in which the composition across the bottom sends an item of fixed point data to the signature of the fixed set mod $2^{\varphi(k)}$. This may be checked on the conjugate Hopf bundle $\eta \rightarrow C P(l)$ by recalling [4] that the resulting value is

$$
\begin{cases}\left(\frac{\lambda+1}{\lambda-1}\right)^{l+1}=x^{l+1} & \text { when } l \text { is even, } \\ 1-\left(\frac{\lambda+1}{\lambda-1}\right)^{l+1}=1-x^{l+1} & \text { when } l \text { is odd. }\end{cases}
$$




\section{REFERENCES}

1. J. P. Alexander, G. C. Hamrick and J. W. Vick, Bilinear forms and cyclic group actions, Bull. Amer. Math. Soc. 80 (1974), 730-734.

2. M. F. Atiyah and G. B. Segal, The index of elliptic operators. II , Ann. of Math. (2) 87 (1968), 531 -545. MR 38 \# 5244.

3. M. F. Atiyah and I. M. Singer, The index of elliptic operators. I, III, Ann. of Math. (2) 87 (1968), 484-530, 546-604. MR 38 \# 5243; \#5245.

4. P. E. Conner, Seminar on periodic maps, Lecture Notes in Math., vol. 46, Springer-Verlag, Berlin and New York, 1967. MR 36 \#7147.

5. P. E. Conner and E. E. Floyd, Maps of odd period, Ann. of Math. (2) 84 (1966), 132-156. MR 34 \# 3587.

Department of Mathematics, University of Texas, Austin, Texas 78712 